\title{
ON THE RESTRICTION OF THE FOURIER TRANSFORM TO A CONICAL SURFACE
}

$\mathrm{BY}$

\author{
BARTOLOME BARCELO TABERNER
}

\begin{abstract}
Let $\Gamma$ be the surface of a circular cone in $\mathbf{R}^{3}$. We show that if $1 \leq p<4 / 3,1 / q=3(1-1 / p)$ and $f \in L^{p}\left(\mathbf{R}^{3}\right)$, then the Fourier transform of $f$ belongs to $L^{q}(\Gamma, d \sigma)$ for a certain natural measure $\sigma$ on $\Gamma$. Following P. Tomas we also establish bounds for restrictions of Fourier transforms to conic annuli at the endpoint $p=4 / 3$, with logarithmic growth of the bound as the thickness of the annulus tends to zero.
\end{abstract}

1. Introduction. Given a conical surface $\Gamma$ in $\mathbf{R}^{3}$ and $f \in L^{p}\left(\mathbf{R}^{3}\right), 1 \leq p<4 / 3$, we prove that we can restrict the Fourier transform of $f, \hat{f}(\xi)$ to the surface of the cone, and we have the bound

$$
\left(\int_{\Gamma}|\hat{f}(\xi)|^{q} d \sigma(\xi)\right)^{1 / q} \leq C_{p, q}\|f\|_{L^{p}\left(\mathbf{R}^{3}\right)}
$$

where $1 / q=3[1-1 / p]$ and $d \sigma(\xi)=r^{-1} d \mu(\xi)$, with $d \mu$ the Lebesgue measure over $\Gamma$ and $r$ the distance to the cone vertex.

This measure is a necessary condition as seen by homogeneity arguments. The homogeneity of the measure absorbs that of the Fourier transform.

Observe that in a compact piece of $\Gamma, d \sigma$ is like the Lebesgue measure.

In an unpublished work, A. Córdoba and E. Stein proved that such inequality is true for $q=2$ in $\mathbf{R}^{n}$. R. Strichartz [5] later extended the result to more general quadratic surfaces. Their proof is done by estimating the Fourier transform of the singular measure over the surface and by using a complex interpolation argument. The conjecture, however, was that the indices $p$ and $q$, for which (1) holds true, are $1 \leq p<4 / 3,1 / q=3[1-1 / p]$.

The idea of the proof consists of desingularising the measure and making $f$ a step function, in order to continue with geometrical arguments in a good decomposition that will allow us to prove the dual bound for (1). The present work follows, in a certain sense, the line introduced by A. Córdoba (see for example $[\mathbf{1}]$ ) and later followed by A. Ruiz [3].

In the third section we go on to prove that for $p=4 / 3$ such an inequality is not possible, but it has a logarithmical growth in the following sense: if the surface of the cone is expanded a little in a homogeneous way, that is, at a certain height $r$, it has a thickness $\delta r$, and if we call the "fat" cone obtained in this way, $\Gamma^{*}$, we then have

$$
\left(\frac{1}{\delta} \int_{\Gamma^{*}}|\hat{f}(\xi)|^{4 / 3} \frac{d \xi}{|\xi|^{2}}\right)^{3 / 4} \leq C|\log \delta|^{1 / 2} \cdot\|f\|_{4 / 3} .
$$

Received by the editors May 10, 1983 and, in revised form, March 1, 1984.

1980 Mathematics Subject Classification. Primary 42B99; Secondary 53A05, 35A22. 
Observe that, if the factor $|\log \delta|$ did not exist on the right-hand side of $(2)$, we could obtain the inequality ( 1 ) by letting $\delta$ go to zero for $p=q=4 / 3$. This is the reason why we affirm that there is a logarithmical growth of the restriction.

To prove (2), we follow $\mathrm{P}$. Tomas [7] who proves the same result for the boundary of the unit circle. To do this, if $\varphi_{\delta}(\xi)$ is the characteristic function of $\Gamma^{*}$ and we set

$$
\psi_{\delta}(\xi)=\frac{1}{|\xi|^{3 / 2}} \varphi_{\delta}(\xi)
$$

(2) can be written as

$$
\left\|\psi_{\delta} \hat{f}\right\|_{4 / 3} \leq C \delta^{3 / 4}|\log \delta|^{1 / 2}\|f\|_{4 / 3}
$$

and, by duality, if we define the multiplier $\left(T_{\delta} f\right)^{\widehat{\Upsilon}}(\xi)=\psi_{\delta}(\xi) \cdot \hat{f}(\xi),(3)$ is equivalent to

$$
\left\|T_{\delta} \hat{f}\right\|_{4} \leq C \delta^{3 / 4}|\log \delta|^{1 / 2}\|f\|_{4},
$$

which is what we prove in Theorem 2 .

Different constants may appear in the course of the proof and these will be denoted by the same letter $C$. When we say that a family of sets has a disjoint intersection, or that it has a finite overlapping, we mean that the number of sets that contain a given point is bound by an absolute constant that is independent of the decomposition. Consequently, the bound obtained will only be changed by a constant factor.

On the other hand, these results are sharp in the sense that Theorem 1 is false if $p>4 / 3$ or $1 / q \neq 3[1-1 / p]$. Also, there is inevitably a power of $|\log \delta|$ on the right-hand side of the inequality of Theorem 2 .

Finally, I am deeply grateful to Professor A. Córdoba for his constant encouragement and advice regarding these problems.

2. Let $\Gamma$ be a conical surface in $\mathbf{R}^{3}$ and let $d \sigma$ be the singular measure introduced before.

THEOREM 1. We have the a priori inequality

$$
\left(\int_{\Gamma}|\hat{f}(\xi)|^{q} d \sigma(\xi)\right)^{1 / q} \leq C_{p, q}\|f\|_{p}
$$

for every $f \in S\left(\mathbf{R}^{3}\right)$ and $1 \leq p<4 / 3,1 / 3=3[1-1 / p]$.

PROOF. Due to the fact that the Fourier transform is well behaved with respect to translations and rotations, we may assume, without loss of generality, that the cone has its vertex at the origin and that it is generated by the half-line $z=x$, $z>0$, around the $z$-axis. We then have the cartesian equation $z^{2}=x^{2}+y^{2}, z>0$, and we can parametrise it by

$$
z=r, \quad x=r \cos \theta, \quad y=r \sin \theta,
$$

and with this parametrisation $d \sigma$ is $d \sigma=d r d \theta$.

It is sufficient to consider the first quadrant $0 \leq \theta \leq \pi / 2$, since the symmetry of the cone will allow us to treat the other cases equally and the result will only be affected by a different constant. We divide the cone into dyadic pieces $\Gamma_{n}, n \in \mathbf{Z}$, 

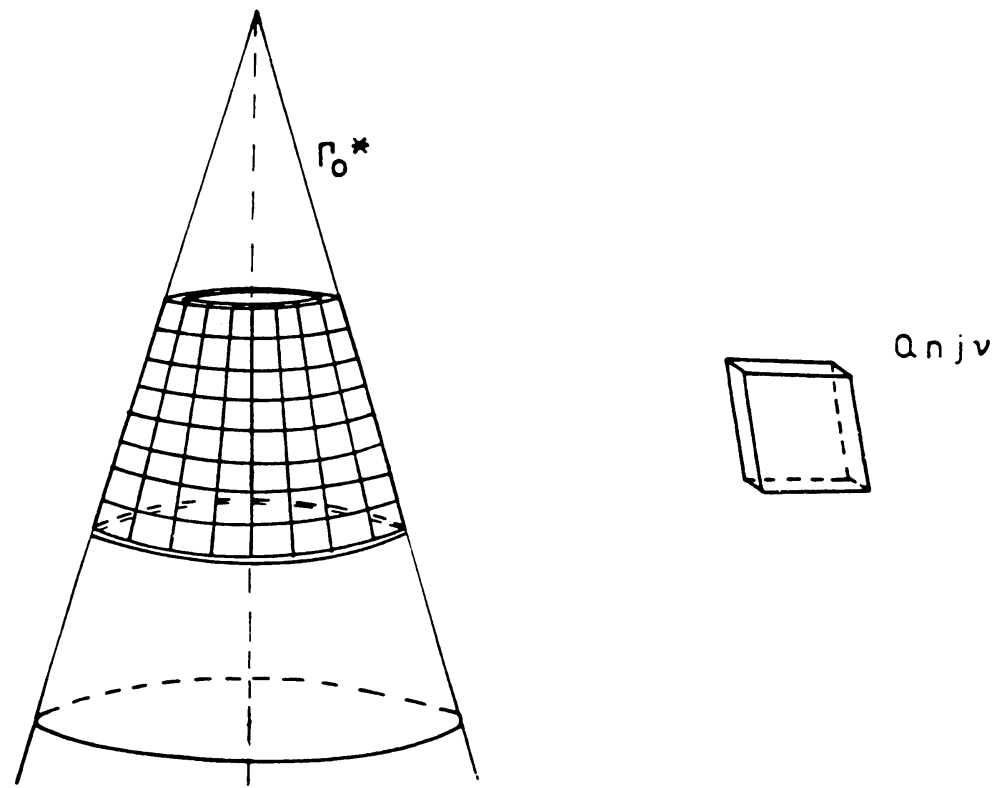

\section{FIGURE 1}

where $\Gamma_{n}$ is the part of the cone comprised between the planes of height $2^{n}$ and $2^{n+1}$.

Given that $\delta>0$, we expand $\Gamma_{0}$ in the radial direction by a thickness $\delta r$, in such a way that we obtain a "fat" cone $\Gamma_{0}^{*}$, and at a height $z$ with $1 \leq z \leq 2$ we obtain the ring $0 \leq \theta \leq \pi / 2, z(1-\delta / 2) \leq r \leq z(1+\delta / 2)$.

We now divide $\Gamma_{0}^{*}$ into $\delta^{-1 / 2} \times \delta^{-1 / 2}$ tiles $Q_{0 j \nu}$, where $Q_{0 j \nu}$ will be the tile of dimensions $\delta \times \delta^{1 / 2} \times \delta^{1 / 2}$ formed by the points of $\Gamma_{0}^{*}$ at a height $1+j \delta^{1 / 2} \leq z \leq$ $1+(j+1) \delta^{1 / 2}$ and with an angular direction $\delta^{1 / 2} \nu \leq \theta \leq \delta^{1 / 2}(\nu+1)$.

Once we obtain the decomposition of $\Gamma_{0}^{*}$, we obtain $\Gamma_{n}^{*}$ and, by homogeneity, a similar partition of $\Gamma_{n}^{*}$ into tiles. In this way, $\Gamma_{n}^{*}$ will be divided into $\delta^{-1 / 2} \times \delta^{-1 / 2}$ pieces $Q_{n j \nu}$, where $Q_{n j \nu}$ is the tile of dimensions $2^{n} \delta \times 2^{n} \delta^{1 / 2} \times 2^{n} \delta^{1 / 2}$ at a height $2^{n}+2^{n} j \delta^{1 / 2} \leq z \leq 2^{n}+2^{n}(j+1) \delta^{1 / 2}$ and an angular direction $\delta^{1 / 2} \nu \leq \theta \leq$ $\delta^{1 / 2}(\nu+1)$. For purposes of calculations, we may assume that $Q_{n j \nu}$ are rectangular tiles adapted to the surface.

We set $\Gamma^{*}=\bigcup_{n} \Gamma_{n}^{*}$ and we call $\varphi_{n j \nu}$ the characteristic function of $Q_{n j \nu}$.

To prove inequality (4), in desingularising the measure, it is sufficient to consider

$$
\lim _{\delta \rightarrow 0}\left(\frac{1}{\delta} \int_{\Gamma^{*}}|\hat{f}(\xi)|^{q} \frac{d \xi}{|\xi|^{2}}\right)^{1 / q} \leq C\|f\|_{p}
$$

and to prove this inequality by duality, it is enough to take functions $g(\xi)=$ $\sum_{n j \nu} a_{n j \nu} \varphi_{n j \nu}(\xi)$ that are constant in each tile, so that for each sufficiently small $\delta>0$

$$
\left\|\sum_{n j \nu} \frac{a_{n j \nu}}{2^{2 n / q}} \hat{\varphi}_{n j \nu}\right\|_{p^{\prime}} \leq C \delta^{1 / q}\left\|\sum_{n j \nu} a_{n j \nu} \varphi_{n j \nu}\right\|_{q^{\prime}}
$$

where $p^{\prime}$ and $q^{\prime}$ are the dual exponents for $p$ and $q$ respectively. 
Because only the limiting case $\delta \rightarrow 0$ is of interest, we consider any fixed portion $2^{-M} \leq z \leq 2^{M}$ of the cone, and we shall prove (5) for $M$ fixed with bounds independent of $M$ once $\delta$ is sufficiently small.

We take then $a_{n j \nu}=0$ for $|n|>M$. We consider also $\delta$ such that

$$
|n-m| \leq\left|\log _{2} \delta\right| / 2
$$

because this geometrically means, if for example $m \leq n$, the diameter of $\Gamma_{m}^{*}$ is smaller than each tile in $\Gamma_{n}^{*}$. This is necessary for the computations that follow.

Since $p^{\prime}>4$, we can apply the Hausdorff-Young theorem to the left-hand side of (5), with $1 / s+2 / p^{\prime}=1$; then

$$
\begin{aligned}
& \left(\int\left|\sum_{n j \nu} \frac{a_{j n \nu}}{2^{2 n / q}} \hat{\varphi}_{n j \nu}(\xi)\right|^{p^{\prime}} d \xi\right)^{1 / p^{\prime}} \\
= & \left(\int\left|\sum_{n m} \sum_{j k} \sum_{\nu \mu} \frac{a_{j n \nu} a_{m k \mu}}{2^{2 n / q} 2^{2 m / q}} \hat{\varphi}_{n j \nu}(\xi) \cdot \hat{\varphi}_{m k \mu}(\xi)\right|^{p^{\prime} / 2} d \xi\right)^{2 / p^{\prime} \cdot 1 / 2} \\
\leq & \left.\left.\left(\int\left|\sum_{n m} \sum_{j k} \sum_{\nu \mu} \frac{a_{n j \nu} a_{m k \mu}}{2^{2 n / q} 2^{2 m / q}} \varphi_{n j \nu} * \varphi_{m k \mu}(x)\right|^{s} d x\right)^{s / 2 s}\right|^{s} d x\right)^{1 / 2 s} \\
\leq & C\left[\int\left|\sum_{m \leq n} \sum_{j k} \sum_{\nu \mu} \frac{a_{n j \nu} a_{m k \mu}}{2^{2 n / q} 2^{2 m / q}} \varphi_{n j \nu} * \varphi_{m k \mu}(x)\right|^{1 / s}\right]^{1 / 2} \\
\leq & {\left[\sum_{r=n-m \geq 0}\left(\int\left|\sum_{n} \sum_{j k} \sum_{\nu \mu} \frac{a_{n j \nu} a_{m k \mu}}{2^{2 n / q} 2^{2 m / q}} \varphi_{n j \nu} * \varphi_{m k \mu}(x)\right|^{s} d x\right)^{1 / 2}\right.} \\
= & C\left[\sum_{r \geq 0} A_{r}\right]^{1 / 2}
\end{aligned}
$$

where, for fixed $r \geq 0, A_{r}$ is the above expression with parentheses in which, once $n$ is taken, $m$ is $m=n-r$.

Note also that $r \leq\left|\log _{2} \delta\right| / 2$ because we are in the fixed portion $2^{-M} \leq z \leq 2^{M}$ of the cone.

The proof follows by carefully analyzing the different terms inside the integrand. To do this we need to estimate the size of the supports and how they overlap.

The role of the $z$-coordinate appears in the vertical overlapping, while the horizontal one is used to study the convolution of a measure $f(\theta) d \theta$ on circles of distinct radii living in $\mathbf{R}^{2}$.

To estimate $A_{r}$, as we shall see in the calculations below, if $m$ is close to $n$, the overlapping is small and the size is large. But if $m$ moves away from $n$, the overlapping increases and the size decreases. It is marvelous to observe that one exactly compensates the other. 
To measure the vertical overlapping, if $m \leq n$, we consider in $\Gamma_{m}^{*}$ the $2^{m} / \delta^{1 / 2} 2^{n}$ rings $\Gamma_{m l}^{*}$, where $\Gamma_{m l}^{*}$ is the ring of height $\delta^{1 / 2} 2^{n}$ obtained by the union $\bigcup_{k, \mu} Q_{m k \mu^{\prime}}$, with $\mu$ varying over all angular directions and $k$ such that

$$
\delta^{1 / 2} 2^{n} l \leq \delta^{1 / 2} 2^{m} k \leq \delta^{1 / 2} 2^{n}(l+1) .
$$

Hence

$$
\left[\Gamma_{m l}^{*}+\operatorname{supp}\left(\varphi_{n j \nu}\right)\right] \cap\left[\Gamma_{m l^{\prime}}+\operatorname{supp}\left(\varphi_{n j^{\prime} \nu^{\prime}}\right)\right] \neq \varnothing
$$

for $l+j=l^{\prime}+j^{\prime}$, while this intersection has a finite overlapping independent of $\delta$ if $l+j \neq l^{\prime}+j^{\prime}$.

Thereby, fixing $n, m$, a point in $\Gamma_{m l}^{*}+\operatorname{supp}\left(\varphi_{n j \nu}\right)$ belongs at most to $2^{m} / \delta^{1 / 2} 2^{n}$ different sums of this kind.

Since

$$
\operatorname{supp}\left(\varphi_{n j \nu} * \sum_{\mu} \varphi_{m k \mu}\right) \cap \operatorname{supp}\left(\varphi_{n j \nu} * \sum_{\mu} \varphi_{m k^{\prime} \mu}\right) \neq \varnothing
$$

for all $k, k^{\prime}$ with $\operatorname{supp}\left(\sum_{\mu} \varphi_{m k \mu}\right) \subset \Gamma_{m l}^{*}$ and $\operatorname{supp}\left(\sum_{\mu} \varphi_{m k^{\prime} \mu}\right) \subset \Gamma_{m l}^{*}$ and there are $2^{n} / 2^{m}$. of these indices, it results that a point in $\operatorname{supp}\left(\varphi_{n j \nu} * \sum_{\mu} \varphi_{m k \mu}\right)$ belongs at most to

$$
\left(2^{m} / \delta^{1 / 2} 2^{n}\right) \cdot\left(2^{n} / 2^{m}\right)=\delta^{-1 / 2}
$$

different supports of this class.

This provides us with the vertical overlapping. We therefore have (7)

$$
\begin{aligned}
& \sum_{r} A_{r}= \sum_{\substack{0 \leq r \leq d / 2\left|\log _{2} \delta\right| \\
r=n-m}}\left\{\int\left|\sum_{n} \sum_{j k} \sum_{\nu \mu} \frac{a_{n j \nu} a_{m k \mu}}{2^{2 n / q} 2^{2 m / q}} \varphi_{n j \nu} * \varphi_{m k \mu}(x)\right|^{s} d x\right\}^{1 / s} \\
& \leq C \sum_{\substack{n-m \leq\left|\log _{2} \delta\right| \\
m \leq n}}\left\{\sum_{n} \sum_{j k} \frac{\delta^{-(s-1) / 2}}{2^{2 n s / q} 2^{2 m s / q}} \mid \sum_{\nu} a_{n j \nu} \varphi_{n j \nu}\right. \\
&\left.\left.* \sum_{\mu} a_{m k \mu} \varphi_{m k \mu}(x)\right|^{s} d x\right\}^{1 / s} .
\end{aligned}
$$

Let us now go on to estimate the horizontal overlapping. We have to bear in mind that if two supports $Q_{n j \nu}$ and $Q_{m k \mu}$ have small angular separation $|\nu-\mu|$, then the size of the sum $Q_{n j \nu}+Q_{m k \mu}$ is going to be as large as $Q_{n j \nu}$ and there will be considerable overlapping with other supports. The contrary happens if there is a large angular separation.

Once we have fixed the heights $j$ and $k$, in order to study the situation it is sufficient to observe how it works in a section $S_{j k}$ perpendicular to the cone axis at a height

$$
2^{n}+2^{m}+j \delta^{1 / 2} 2^{n}+k \delta^{1 / 2} 2^{m}
$$

because $\operatorname{supp}\left(\varphi_{n j \nu} * \sum_{\mu} \varphi_{m k \mu}\right)$ is found at this height and the final sum $Q_{n j \nu}+$ $Q_{m k \mu}$ would be obtained by moving its section with $S_{j k}$ parallel to the vertical direction of $Q_{n j \nu}$. 


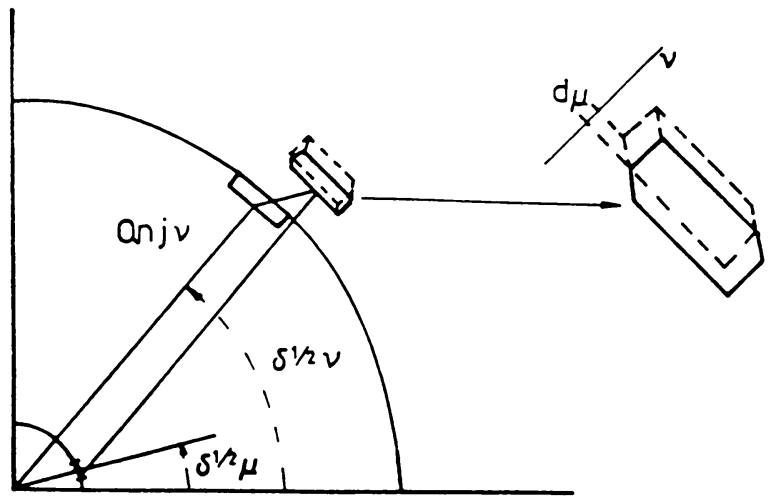

FIGURE 2

In the $S_{j k}$ plane, $\operatorname{supp}\left(\varphi_{n j \nu} * \varphi_{m k \mu}\right)$ is centered at the point

$$
2^{n}\left(1+\delta^{1 / 2} j\right) e^{\pi \nu i / 2}+2^{m}\left(1+\delta^{1 / 2} k\right) e^{\pi \mu i / 2}
$$

and is essentially like a block of length $2^{n} \delta^{1 / 2}$ and width

$$
\delta^{1 / 2} 2^{n}+\delta^{1 / 2} 2^{m} \sin \left((|\nu-\mu|+1) \delta^{1 / 2}\right) .
$$

Also, $\operatorname{supp}\left(\varphi_{n j \nu} * \varphi_{m k \mu}\right)$ is translated a distance $\delta^{1 / 2} 2^{m} \sin \left(|\nu-\mu| \delta^{1 / 2}\right) \simeq$ $\delta 2^{m}|\nu-\mu|$ from $\operatorname{supp}\left(\varphi_{n j \nu} * \varphi_{m, k, \mu+1}\right)$, as is observed if it is projected in the $\nu$ direction.

To measure the number of $\operatorname{sums} \operatorname{supp}\left(\varphi_{n j \nu} * \varphi_{m k \mu}\right)$ that overlap, we will distinguish the following two cases:

(i) $|\nu-\mu|+1 \leq 2^{n-m}$.

In this case a point belongs to no more than $N$ different supports, with $N$ such that

$$
\delta 2^{m}|\nu-\mu|+\delta 2^{m}(|\nu-\mu|-1)+\cdots+\delta 2^{m}(|\nu-\mu|-N) \simeq \delta 2^{n},
$$

that is $N \leq 2^{n-m} /(|\nu-\mu|+1)$. Moreover,

$$
\left|\operatorname{supp}\left(\varphi_{n j \nu} * \varphi_{m k \mu}\right)\right| \simeq\left|\operatorname{supp}\left(\varphi_{n j \nu}\right)\right|=\delta^{2} 2^{3 n}
$$

and

$$
\left\|\varphi_{n j \nu} * \varphi_{m k \mu}\right\|_{\infty} \simeq\left|\operatorname{supp}\left(\varphi_{m k \mu}\right)\right|=\delta^{2} 2^{3 m}
$$

hence $\int\left|\varphi_{n j \nu} * \varphi_{m k \mu}(x)\right|^{s} d x \leq C \delta^{2 s+2} 2^{3 m s} 2^{3 n}$.

(ii) $|\nu-\mu|+1 \geq 2^{n-m}$.

Then $\operatorname{supp}\left(\varphi_{n j \nu} * \varphi_{m k \mu}\right)$ has a finite overlapping when $\mu$ varies, and

$$
\begin{aligned}
\left|\operatorname{supp}\left(\varphi_{n j \nu} * \varphi_{m k \mu}\right)\right| & \leq C \delta^{2} 2^{2 n} 2^{m}(|\nu-\mu|+1) \\
\left\|\varphi_{n j \nu} * \varphi_{m k \mu}\right\|_{\infty} & \leq C \delta^{2} 2^{n} 2^{2 m} /(|\nu-\mu|+1)
\end{aligned}
$$

Therefore,

$$
\int\left|\varphi_{n j \nu} * \varphi_{m k \mu}(x)\right|^{s} d x \leq C \frac{\delta^{2 s+2} 2^{n(2+s)} 2^{m(2 s+1)}}{(|\nu-\mu|+1)^{s-1}}
$$


Then with (i) and (ii) we can write

$$
\begin{aligned}
& \text { (7) } \leq C \sum_{\substack{n-m \leq\left|\log _{2} \delta\right| \\
m \leq n}}\left\{\sum_{n} \sum_{j k} \frac{\delta^{-(s-1) / 2}}{2^{2 n s / q} 2^{2 m s / q}}\right. \\
& \cdot\left[\sum_{|\nu-\mu|+1 \leq 2^{n-m}}\left(\frac{2^{n-m}}{|\nu-\mu|+1}\right)^{s-1} \cdot \delta^{2 s+2} 2^{3 n} 2^{3 m s}\left|a_{n j \nu}\right|^{s}\left|a_{m k \mu}\right|^{s}\right. \\
& \left.\left.+\sum_{|\nu-\mu|+1 \geq 2^{n-m}} \delta^{2 s+2} 2^{n(2+s)} 2^{m(2 s+1)} \frac{\left|a_{n j \nu}\right| s\left|a_{m k \mu}\right|^{s}}{(|\nu-\mu|+1)^{s-1}}\right]\right\}^{1 / s} \\
& \leq C \sum_{\substack{n-m \leq\left|\log _{2} \delta\right| \\
m \leq n}}\left\{\sum_{n} \sum_{j k} \frac{\delta^{-(s-1) / 2} \delta^{2 s+2} 2^{m(2 s+1)} 2^{n(2+s)}}{2^{2 n s / q} 2^{2 m s / q}}\right. \\
& \left.\cdot \sum_{\nu \mu} \frac{\left|a_{n j \nu}\right|^{s}\left|a_{m k \mu}\right|^{s}}{(|\nu-\mu|+1)^{s-1}}\right\}^{1 / s}
\end{aligned}
$$

and by a fractional integration argument

$$
\begin{aligned}
& \sum_{\nu \mu} \frac{\left|a_{n j \nu}\right|^{s}\left|a_{m k \mu}\right|^{s}}{(|\nu-\mu|+1)^{s-1}} \\
& \quad \leq C\left(\sum_{\nu}\left|a_{n j \nu}\right|^{2 s /(3-s)}\right)^{(3-s) / 2}\left(\sum_{\mu}\left|a_{m k \mu}\right|^{2 s /(3-s)}\right)^{(3-s) / 2} ;
\end{aligned}
$$

hence

$$
\begin{aligned}
&(7) \leq C \sum_{\substack{n-m \leq\left|\log _{2} \delta\right| \\
m \leq n}} \delta^{(3 s-3) / s} 2^{-|n-m|(s-1) / s} \\
& \cdot\left\{\sum_{n}\left(\sum_{j \nu}\left|a_{n j \nu}\right|^{2 s /(3-s)} \delta^{2} 2^{3 n}\right)^{(3-s) / 2}\right. \\
&\left.\cdot\left(\sum_{k \mu}\left|a_{m k \mu}\right|^{2 s /(3-s)} \delta^{2} 2^{3 m}\right)^{(3-s) / 2}\right\}^{1 / s} \\
& \leq C \delta^{(3 s-3) / s}\left(\sum_{\substack{n-m \leq\left|\log _{2} \delta\right| \\
m \leq n}} 2^{-|n-m|(s-1) / s}\right)\left(\sum_{n j \nu}\left|a_{n j \nu}\right|^{2 s /(3-s)} \delta^{2} 2^{3 n}\right)^{(3-s) / s}
\end{aligned}
$$

but

and then we have proved

$$
\sum_{\substack{n-m \leq\left|\log _{2} \delta\right| \\ m \leq n}} 2^{-|n-m|(s-1) / s} \leq C,
$$

$$
\sum_{r \geq 0} A_{r} \leq C \delta^{(3 s-3) / s}\left(\sum_{n j \nu}\left|a_{n j \nu}\right|^{2 s /(3-s)} \delta^{2} 2^{3 n}\right)^{(3-s) / s} .
$$


Now, observing that $(3 s-3) / 2 s=1 / q,(3-s) / 2 s=1 / q^{\prime}$, we can conclude finally

$$
\left\|\sum_{n j \nu} \frac{a_{n j \nu}}{2^{2 n / q}} \hat{\varphi}_{n j \nu}\right\|_{p^{\prime}} \leq C\left(\sum_{r} A_{r}\right)^{1 / 2} \leq C \delta^{1 / q}\left\|\sum_{n j \nu} a_{n j \nu} \varphi_{n j \nu}\right\|_{q^{\prime}},
$$

which is inequality (5).

We notice that in fact we have proved a more general theorem:

Given any strictly convex cone in $\mathbf{R}^{3}$, that is, a surface $\Gamma$ given by $z=r(x, y)$ where $r(x, y)>0$ is homogeneous of degree 1 on $\mathbf{R}^{2}$ with $\{(x, y) \mid r(x, y)=1\}$ having positive curvature at each point, if $d \sigma$ is the measure $d \sigma=r^{\beta} d \mu$ where $-2 \leq \beta \leq-1$ and $d \mu$ the Lebesgue measure over $\Gamma$, then we have

$$
\|\hat{f}\|_{L^{q}(\Gamma, d \sigma)} \leq C_{p, q}\|f\|_{L^{p}\left(\mathbf{R}^{3}\right)} \quad \text { where } p^{\prime}>2 \frac{1-\beta}{\beta+2} \text { and } q=\frac{2+\beta}{3} p^{\prime} .
$$

The proof is the same as in Theorem 1 introducing the straightforward changes. The result is sharp as we can see using homogeneity arguments.

3. Using the decomposition and the notation of the previous section, let $\psi_{\delta}(\xi)$ be the characteristic function of the "fat" cone widened by a thickness $\delta r, \Gamma^{*}$, and let

$$
\psi_{\delta}(\xi)=\frac{1}{|\xi|^{3 / 2}} \varphi_{\delta}(\xi)
$$

Then we define the multiplier

$$
\left(T_{\delta} f\right)^{\uparrow}(\xi)=\psi_{\delta}(\xi) \cdot \hat{f}(\xi) .
$$

THEOREM 2. There exists a constant $C$, independent of $\delta$, such that

$$
\left\|T_{\delta} \hat{f}\right\|_{4} \leq C \delta^{3 / 4}|\log \delta|^{1 / 2}\|f\|_{4}
$$

for every $f \in S\left(\mathbf{R}^{3}\right)$.

PROOF. If $\varphi_{\delta}^{n}(\xi)$ is the characteristic function of the dyadic piece of the cone $\Gamma_{n}^{*}$, we set $\psi_{\delta}^{n}(\xi)=\varphi_{\delta}^{n}(\xi) \cdot \psi_{\delta}(\xi)$ and let $S_{n}$ be the multiplier $\left(S_{n} f\right)^{\curlyvee}(\xi)=\psi_{\delta}^{n}(\xi) \cdot \hat{f}(\xi)$.

By the Littlewood-Paley theorem $\left\|T_{\delta} \hat{f}\right\|_{4} \simeq\left\|\left(\sum_{n}\left|S_{n} \hat{f}\right|^{2}\right)^{1 / 2}\right\|_{4}$, which implies

$$
\begin{aligned}
\left\|T_{\delta} \hat{f}\right\|_{4}^{4} & \simeq\left\|\left(\sum_{n}\left|S_{n} \hat{f}\right|^{2}\right)^{1 / 2}\right\|_{4}^{4}=\int_{\mathbf{R}^{3}}\left(\sum_{n}\left|S_{n} \hat{f}(\xi)\right|^{2}\right)^{2} d \xi \\
& =\sum_{n, m} \int\left|S_{n} \hat{f}(\xi) \cdot S_{m} \hat{f}(\xi)\right|^{2} d \xi=\sum_{n, m} \int\left|\left(S_{n} \hat{f}\right)^{\wedge} *\left(S_{m} \hat{f}\right)^{\uparrow}(x)\right|^{2} d x \\
& \leq C \sum_{m \leq n} \int\left|\sum_{j \nu} \frac{1}{2^{3 n / 2}} f_{n j \nu} * \sum_{k \mu} \frac{1}{2^{3 n / 2}} f_{m k \mu}(x)\right|^{2} d x
\end{aligned}
$$

where $f_{n j \nu}=f \cdot \varphi_{n j \nu}$ is the restriction of $f$ to the tile $Q_{n j \nu}$, which is in $\Gamma_{n}^{*}$ at a height $2^{n}+2^{n} j \delta^{1 / 2}$ and an angular direction $\delta^{1 / 2} 2^{n} \nu$. 
We now divide sum (9) into $\left|\log _{2} \delta\right|$ blocks of $n, m$ indices, in each of which we have $n-m \geq\left|\log _{2} \delta\right|$. Let $\Delta$ be one of these; then

(10)

$$
\begin{aligned}
(9) & \leq C\left|\log _{2} \delta\right| \sum_{\substack{n, m \in \Delta \\
m \leq n}} \int\left|\sum_{j \nu} \frac{1}{2^{3 n / 2}} f_{n j \nu} * \sum_{k \mu} \frac{1}{2^{3 m / 2}} f_{m k \mu}(x)\right|^{2} d x \\
& \leq C|\log \delta|\left(\sum_{n \in \Delta} \int\left|\sum_{j \nu} \frac{1}{2^{3 n / 2}} f_{n j \nu} * \sum_{k \mu} \frac{1}{2^{3 m / 2}} f_{m k \mu}(x)\right|^{2} d x\right. \\
& \left.+\sum_{\substack{n, m \in \Delta \\
m<n}} \int\left|\sum_{j \nu} \frac{1}{2^{3 n / 2}} f_{n j \nu} * \sum_{k \mu} \frac{1}{2^{3 m / 2}} f_{m k \mu}(x)\right|^{2} d x\right) \\
= & C|\log \delta|\left(\sum_{(1)}+\sum_{(2)}\right) .
\end{aligned}
$$

(a) To estimate $\sum_{(1)}$ we observe that

$$
\operatorname{supp}\left(\sum_{\nu} f_{n j \nu} * \sum_{\mu} f_{n k \mu}\right) \cap \operatorname{supp}\left(\sum_{\nu} f_{n j^{\prime} \nu} * \sum_{\mu} f_{n k^{\prime} \mu}\right) \neq \varnothing
$$

if and only if $\left|j-j^{\prime}\right|+\left|k-k^{\prime}\right| \leq C$, where $C$ is independent of $\delta$.

As $j, k$ vary from 1 to $\delta^{-1 / 2}$; we have that $\operatorname{supp}\left(\sum_{\nu} f_{n j \nu} * \sum_{\mu} f_{n k \mu}\right)$ has a maximal overlapping with other supports of $\delta^{-1 / 2}$, and in this way

$$
\begin{aligned}
\sum_{(1)} & =\sum_{n \in \Delta} \int\left|\sum_{j \nu} \frac{1}{2^{3 n / 2}} f_{n j \nu} * \sum_{k \mu} \frac{1}{2^{3 n / 2}} f_{n k \mu}(x)\right|^{2} d x \\
& \leq C \sum_{n} \frac{\delta^{-1 / 2}}{2^{6 n}} \sum_{j k} \int\left|\sum_{\nu} f_{n j \nu} * \sum_{\mu} f_{n k \mu}(x)\right|^{2} d x \\
& \leq C \sum_{n} \frac{\delta^{-1 / 2}}{2^{6 n}} \sum_{j k} \sum_{\nu \mu} \int\left|f_{n j \nu} * f_{n k \mu}(x)\right|^{2} d x
\end{aligned}
$$

since

$$
\operatorname{supp}\left(f_{n j \nu} * f_{n k \mu}\right) \cap \operatorname{supp}\left(f_{n j \nu^{\prime}} * f_{n k \mu^{\prime}}\right)=\varnothing
$$

if $(\nu, \mu) \neq\left(\nu^{\prime}, \mu^{\prime}\right)$.

LEMMA 1.

$$
\int\left|f_{n j \nu} * f_{n k \mu}(x)\right|^{2} d x \leq C \frac{\delta^{2} 2^{3 n}}{|\nu-\mu|+1}\left\|f_{n j \nu}\right\|_{2}^{2}\left\|f_{n k \mu}\right\|_{2}^{2} .
$$

Proof. By the Cauchy-Schwarz inequality

$$
\left|f_{n j \nu} * f_{n k \mu}(x)\right|^{2} \leq\left(\left|f_{n j \nu}\right|^{2} *\left|f_{n k \mu}\right|^{2}\right)(x) \cdot\left(\chi_{Q_{n j \nu}} * \chi_{Q_{n k \mu}}\right)(x) \text {. }
$$


Then integrating this inequality and using

$$
\left\|\chi_{Q_{n j \nu}} * \chi_{Q_{n k \mu}}\right\|_{\infty} \leq C \delta^{2} 2^{3 n}(|\nu-\mu|+1)^{-1}
$$

we obtain (11).

Given the lemma we have

$$
\begin{aligned}
\sum_{(1)} & \leq C \sum_{n} \frac{\delta^{-1 / 2}}{2^{6 n}} \delta^{2} 2^{3 n} \sum_{j k} \sum_{\nu \mu} \frac{\left\|f_{n j \nu}\right\|_{2}^{2}\left\|f_{n k \mu}\right\|_{2}^{2}}{|\nu-\mu|+1} \\
& \leq C \delta^{3 / 2} \sum_{n} \frac{1}{2^{3 n}} \sum_{j k}|\log \delta|\left(\sum_{\nu}\left\|f_{n j \nu}\right\|_{2}^{4}\right)^{1 / 2} \cdot\left(\sum_{\mu}\left\|f_{n k \mu}\right\|_{2}^{4}\right)^{1 / 2} \\
& \leq C \delta^{3 / 2} \sum_{n} \frac{1}{2^{3 n}}|\log \delta|\left(\delta^{-1 / 2}\right)^{(1 / 2) \cdot 2}\left(\sum_{j \nu}\left\|f_{n j \nu}\right\|_{2}^{4}\right)^{1 / 2} \cdot\left(\sum_{k \mu}\left\|f_{n k \mu}\right\|_{2}^{4}\right)^{1 / 2}
\end{aligned}
$$

and since

$$
\left\|f_{n j \nu}\right\|_{2}^{2}=\int\left|f_{n j \nu}\right|^{2} \leq\left(\delta^{2} 2^{3 n}\right)^{1 / 2}\left\|f_{n j \nu}\right\|_{4}^{2},
$$

we finally obtain

$$
\begin{aligned}
\sum_{(1)} & \leq C \delta|\log \delta| \sum_{n} \delta^{2} \frac{2^{3 n}}{2^{3 n}}\left(\sum_{j \nu}\left\|f_{n j \nu}\right\|_{4}^{4}\right) \\
& =C \delta^{3}|\log \delta| \sum_{n j \nu}\left\|f_{n j \nu}\right\|_{4}^{4}=C \delta^{3}|\log \delta|\|f\|_{4}^{4}
\end{aligned}
$$

(b) We shall now estimate

$$
\sum_{(2)}=\sum_{\substack{n, m \in \Delta \\ n \neq m \\ m \leq n}} \frac{1}{2^{3 n} 2^{3 m}} \int\left|\sum_{j \nu} f_{n j \nu} * \sum_{k \mu} f_{m k \mu}(x)\right|^{2} d x
$$

since $2^{m} \leq \delta 2^{n}$, we have

$$
\operatorname{supp}\left(f_{n j \nu} * \sum_{k \mu} f_{m k \mu}\right) \cap \operatorname{supp}\left(f_{n j^{\prime} \nu^{\prime}} * \sum_{k \mu} f_{m k \mu}\right)=\varnothing
$$

except for $\left|j-j^{\prime}\right|+\left|\nu-\nu^{\prime}\right| \leq C$. 
Thus

$$
\begin{aligned}
& \sum_{(2)} \leq C \sum_{\substack{n, m \in \Delta \\
m<n}} \frac{1}{2^{3 n}} \frac{1}{2^{3 m}} \sum_{j \nu} \int\left|f_{n j \nu} * \sum_{k \mu} f_{m k \mu}(x)\right|^{2} d x \\
& =C \sum_{\substack{n, m \in \Delta \\
m<n}} \frac{1}{2^{3 n}} \frac{1}{2^{3 m}} \sum_{j \nu} \int\left|\hat{f}_{n j \nu}(\xi)\right|^{2} \cdot\left|\sum_{k \mu} \hat{f}_{m k \mu}(\xi)\right|^{2} d \xi \\
& \leq C \int\left(\sum_{n \in \Delta} \sum_{j \nu} \frac{1}{2^{3 n}}\left|\hat{f}_{n j \nu}(\xi)\right|^{2}\right)\left(\sum_{m \in \Delta} \frac{1}{2^{3 m}}\left|\sum_{k \mu} \hat{f}_{m k \mu}(\xi)\right|^{2}\right) d \xi \\
& \leq C\left(\int\left(\sum_{n j \nu} \frac{1}{2^{3 n}}\left|\hat{f}_{n j \nu}(\xi)\right|^{2}\right)^{2} d \xi\right)^{1 / 2} \\
& \cdot\left(\int \sum_{m} \frac{1}{2^{3 m}}\left|\sum_{k \mu} \hat{f}_{m k \mu}(\xi)\right|^{2} d \xi\right)^{1 / 2} \\
& \leq C\left(\sum_{(3)}\right)^{1 / 2}\left(\sum_{(1)}+\sum_{(2)}\right)^{1 / 2}
\end{aligned}
$$

where we have written

$$
\begin{aligned}
\sum_{(3)} & =\int\left(\sum_{n j \nu} \frac{1}{2^{3 n}}\left|\hat{f}_{n j \nu}(\xi)\right|^{2}\right)^{2} d \xi \\
& =\sum_{n, n^{\prime} \in \Delta} \sum_{j j^{\prime}} \sum_{\nu \nu^{\prime}} \frac{1}{2^{3 n}} \frac{1}{2^{3 n^{\prime}}} \int\left|f_{n j \nu} * f_{n^{\prime} j^{\prime} \nu^{\prime}}(x)\right|^{2} d x \\
& =\sum_{(1)}+\sum_{n \neq n^{\prime}} \sum_{j j^{\prime}} \sum_{\nu \nu^{\prime}} \frac{1}{2^{3 n}} \frac{1}{2^{3 n^{\prime}}} \int\left|f_{n j \nu} * f_{n^{\prime} j^{\prime} \nu^{\prime}}(x)\right|^{2} d x
\end{aligned}
$$

and if $n^{\prime} \leq n-\left|\log _{2} \delta\right|$,

$$
\int\left|f_{n j \nu} * f_{n^{\prime} j^{\prime} \nu^{\prime}}(x)\right|^{2} d x \leq C \delta^{2} 2^{3 n^{\prime}}\left\|f_{n j \nu}\right\|_{2}^{2}\left\|f_{n^{\prime} j^{\prime} \nu^{\prime}}\right\|_{2}^{2}
$$

whose proof is like that of Lemma 1. Therefore

$$
\sum_{(3)} \leq \sum_{(1)}+C \sum_{n^{\prime} \leq n} \sum_{j j^{\prime}} \sum_{\nu \nu^{\prime}} \frac{1}{2^{3 n}} \frac{1}{2^{3 n^{\prime}}} \delta^{2} \delta^{3 n^{\prime}}\left\|f_{n j \nu}\right\|_{2}^{2}\left\|f_{n^{\prime} j^{\prime} \nu^{\prime}}\right\|_{2}^{2}
$$


with $\left\|f_{n j \nu}\right\|_{2}^{2} \leq\left|\operatorname{supp}\left(f_{n j \nu}\right)\right|^{1 / 2}\left\|f_{n j \nu}\right\|_{4}^{2}=\left(\delta^{2} 2^{3 n}\right)^{1 / 2}\left\|f_{n j \nu}\right\|_{4}^{2}$. Thus

$$
\begin{aligned}
\sum_{(3)} & \leq \sum_{(1)}+C \sum_{n^{\prime} \leq n} \delta^{4} 2^{-\left|n-n^{\prime}\right| 3 / 2} \sum_{j j^{\prime}} \sum_{\nu \nu^{\prime}}\left\|f_{n j \nu}\right\|_{4}^{2}\left\|f_{n j^{\prime} \nu^{\prime}}\right\|_{4}^{2} \\
& \leq \sum_{(1)}+C \delta^{4} \delta^{3 / 2}\left(\sum_{n}\left(\sum_{j \nu}\left\|f_{n j \nu}\right\|_{4}^{2}\right)^{2}\right)^{1 / 2} \cdot\left(\sum_{n^{\prime}}\left(\sum_{j^{\prime} \nu^{\prime}}\left\|f_{n^{\prime} j^{\prime} \nu^{\prime}}\right\|_{4}^{2}\right)^{2}\right)^{1 / 2} \\
& \leq \sum_{(1)}+C \delta^{4} \delta^{3 / 2} \delta^{-1}\left(\sum_{n j \nu}\left\|f_{n j \nu}\right\|_{4}^{4}\right)^{1 / 2}\left(\sum_{n^{\prime} j^{\prime} \nu^{\prime}}\left\|f_{n^{\prime} j^{\prime} \nu^{\prime}}\right\|_{4}^{4}\right)^{1 / 2} \\
& =\sum_{(1)}+C \delta^{9 / 2}\|f\|_{4}^{4} .
\end{aligned}
$$

Now, from this last inequality and (12) we have

$$
\sum_{(2)} \leq C\left(\sum_{(1)}+\sum_{(2)}\right)^{1 / 2}\left(\sum_{(1)}+C \delta^{9 / 2}\|f\|_{4}^{4}\right)^{1 / 2}
$$

and since from (12)

$$
\sum_{(1)} \leq C \delta^{3}|\log \delta|\|f\|_{4}^{4}
$$

we obtain

$$
\sum_{(1)}+\sum_{(2)} \leq C \delta^{3}|\log \delta|\|f\|_{4}^{4}
$$

Inserting this expression into (11) we finally obtain

$$
\left\|T_{\delta} \hat{f}\right\|_{4} \leq C|\log \delta|^{1 / 2} \delta^{3 / 4}\|f\|_{4},
$$

which is the assertion (8) of Theorem 2.

We notice that if we had refined the proof of Theorem 2 we would obtain

$$
\left\|T_{\delta} \hat{f}\right\|_{4} \leq C \delta^{3 / 4}|\log \delta|^{1 / 4}\|f\|_{4}
$$

for every $f \in L^{4}\left(\mathbf{R}^{3}\right)$.

The equality is satisfied by taking a particular $f$ (see P. Tomas [7]); then there is an inevitable power of $|\log \delta|$ on the right-hand side of inequality (8) and $1 / 4$ is the accurate power of $|\log \delta|$ that must appear.

\section{REFERENCES}

1. A. Cordoba, Euclidean harmonic analysis (University of Maryland, 1979), Lecture Notes in Math., vol. 779, Edited by J. J. Benedetto, Springer-Verlag, 1980, pp. 154-162.

2. E. Prestini, A restriction theorem for space curves, Proc. Amer. Math. Soc. 70 (1978), 8-10.

3. A. Ruiz, On the restriction of Fourier transforms to curves, Proc. Conf. on Harmonic Analysis in honour of Antoni Zygmund, University of Chicago, Vol. I, 1983, pp. 186-211.

4. E. Stein, Some problems in harmonic analysis, Proc. Sympos. Pure Math., vol. 35, Part 1, Amer. Math. Soc., Providence, R.I., 1979.

5. R. Strichartz, Restrictions of Fourier transforms to quadratic surfaces and decay of solutions of wave equations, Duke Math. J. 44 (1977), 705-714. 
6. P. A. Tomas, Restriction theorems for the Fourier transform, Proc. Sympos. Pure Math., Vol. 35, Part 1, Amer. Math. Soc., Providence, R.I., 1979, pp. 111-114.

7. __ A note on restriction, Indiana Univ. Math. J. 29 (1980), 287-292.

8. A. Zygmund, On Fourier coefficients and transforms of functions of two variables, Studia Math. 50 (1974), 189-202.

Division de Matematicas, Universidad Autonoma de Madrid, 28049 Madrid, SPAIN

Current address: School of Mathematics, University of Minnesota, Minneapolis, Minnesota 55455 